\title{
Dynamics of collapsing and exploding Bose-Einstein condensate
}

\author{
Sadhan K. Adhikari \\ Instituto de Física Teórica, Universidade Estadual Paulista, 01.405-900 São Paulo, São Paulo, Brazil
}

(October 30, 2018)

\begin{abstract}
Recently, Donley et al. performed an experiment on the dynamics of collapsing and exploding Bose-Einstein condensates by suddenly changing the scattering length of atomic interaction to a large negative value on a preformed repulsive condensate of ${ }^{85} \mathrm{Rb}$ atoms in an axially symmetric trap. Consequently, the condensate collapses and ejects atoms via explosions. We show that the accurate numerical solution of the time-dependent Gross-Pitaevskii equation with axial symmetry can explain some aspects of the dynamics of the collapsing condensate.
\end{abstract}

Since the successful detection [1 15 ] of Bose-Einstein condensates (BEC) in dilute bosonic atoms employing magnetic trap at ultra-low temperature, one problem of extreme interest is the dynamical study of the formation and decay of BEC for attractive atomic interaction 4,5 .

For attractive interaction the condensate is stable for a maximum critical number $N_{\text {cr }}$ of atoms . When the number of atoms increases beyond this critical number, due to interatomic attraction the radius of BEC tends to zero and the central density tends to infinity. Consequently, the condensate collapses emitting atoms until the number of atoms is reduced below $N_{\mathrm{cr}}$ and a stable configuration is reached. With a supply of atoms from an external source the condensate can grow again and thus a series of collapses can take place, which was observed experimentally in the BEC of ${ }^{7} \mathrm{Li}$ with attractive interaction [4]. Theoretical analyses based on the time-dependent mean-field Gross-Pitaevskii (GP) equation also confirm the collapse [6 11].

It is possible to manipulate the interatomic interaction by an external magnetic field via a Feshbach resonance [12]. Roberts et al. [13] and Cornish et al. 14] (at JILA) exploited this idea to suddenly change the atomic scattering length by a large amount in experiment. They have been able to even change the sign of the scattering length, thus changing a repulsive condensate in to an attractive one and vice versa. Consequently, a stable preformed repulsive condensate is suddenly turned to a highly explosive and collapsing attractive condensate. In a classic experiment performed at JILA Donley et al. [15] studied the dynamics of collapsing and exploding condensates formed of ${ }^{85} \mathrm{Rb}$ atoms. The natural scattering length of ${ }^{85} \mathrm{Rb}$ atoms is negative (attractive). By exploiting the Feshbach resonance they made it positive (repulsive) in the initial state. They have provided a quantitative measurement of this explosion by counting the number of emitted and remaining atoms in the condensate as a function of time until an equilibrium is reached. They also measured the energy distribution of the emitted atoms. They claim that their experiment reveal many interesting phenomena that challenge theoretical models.

We demonstrate that some aspects of the above collapse and explosion of the attractive condensate of ${ }^{85} \mathrm{Rb}$ atoms can be explained from an accurate numerical solution of the GP equation in an axially symmetric trap, where we include a quintic three-body nonlinear recombination term which accounts for the decay of the strongly attractive condensate. The numerical method, we use, for the solution of the time-dependent GP equation with an axially symmetric trap has appeared elsewhere 16 22].

There have been other theoretical studies [6 10] to deal with dynamical collapse including an absorptive term to account for the loss of particles. Instead of attempting a full numerical solution of the GP equation with axial symmetry, these investigations used various approximations to study the time evolution of the condensate or employed a spherically symmetric trap.

The time-dependent Bose-Einstein condensate wave function $\Psi(\mathbf{r} ; \tau)$ at position $\mathbf{r}$ and time $\tau$ may be described by the following mean-field nonlinear GP equation 11]

$$
\left[-\frac{\hbar^{2} \nabla^{2}}{2 m}+V(\mathbf{r})+g N|\Psi(\mathbf{r} ; \tau)|^{2}-i \hbar \frac{\partial}{\partial \tau}\right] \Psi(\mathbf{r} ; \tau)=0
$$

Here $m$ is the mass and $N$ the number of atoms in the condensate, $g=4 \pi \hbar^{2} a / m$ the strength of interatomic interaction, with $a$ the atomic scattering length. The trap potential with cylindrical symmetry may be written as $V(\mathbf{r})=\frac{1}{2} m \omega^{2}\left(r^{2}+\lambda^{2} z^{2}\right)$ where $\omega$ is the angular frequency in the radial direction $r$ and $\lambda \omega$ that in the axial direction $z$. We are using the cylindrical coordinate system $\mathbf{r} \equiv(r, \theta, z)$ with $\theta$ the azimuthal angle. The normalization condition of the wave function is $\int d \mathbf{r}|\Psi(\mathbf{r} ; \tau)|^{2}=1$.

In the absence of angular momentum the wave function has the form $\Psi(\mathbf{r} ; \tau)=\psi(r, z ; \tau)$. Now transforming to dimensionless variables defined by $x=\sqrt{2} r / l, y=\sqrt{2} z / l$, $t=\tau \omega, l \equiv \sqrt{\hbar /(m \omega)}$, and

$$
\phi(x, y ; t) \equiv \frac{\varphi(x, y ; t)}{x}=\sqrt{\frac{l^{3}}{\sqrt{8}}} \psi(r, z ; \tau),
$$

we get

$$
\left[-i \frac{\partial}{\partial t}-\frac{\partial^{2}}{\partial x^{2}}+\frac{1}{x} \frac{\partial}{\partial x}-\frac{\partial^{2}}{\partial y^{2}}+\frac{1}{4}\left(x^{2}+\lambda^{2} y^{2}-\frac{4}{x^{2}}\right)\right.
$$




$$
\left.+8 \sqrt{2} \pi n\left|\frac{\varphi(x, y ; t)}{x}\right|^{2}\right] \varphi(x, y ; t)=0
$$

where $n=N a / l$. The normalization condition of the wave function becomes

$$
\mathcal{N}_{\text {norm }} \equiv 2 \pi \int_{0}^{\infty} d x \int_{-\infty}^{\infty} d y|\varphi(x, y ; t)|^{2} x^{-1}=1 .
$$

The root mean square (rms) sizes $x_{\mathrm{rms}}$ and $y_{\mathrm{rms}}$ are defined by

$$
\begin{gathered}
x_{\mathrm{rms}}^{2}=\mathcal{N}_{\mathrm{norm}}^{-1} 2 \pi \int_{0}^{\infty} d x \int_{-\infty}^{\infty} d y|\varphi(x, y ; t)|^{2} x, \\
y_{\mathrm{rms}}^{2}=\mathcal{N}_{\mathrm{norm}}^{-1} 2 \pi \int_{0}^{\infty} d x \int_{-\infty}^{\infty} d y|\varphi(x, y ; t)|^{2} y^{2} x^{-1} .
\end{gathered}
$$

It is now appropriate to calculate the parameters of the present dimensionless GP equation (3) corresponding to the experiment at JILA |15]. We follow the notation and nomenclature of Ref. 15]. Their radial and axial trap frequencies are $\nu_{\text {radial }}=17.5 \mathrm{~Hz}$ and $\nu_{\text {axial }}=6.8 \mathrm{~Hz}$, respectively, leading to $\lambda=0.389$. The harmonic oscillator length $l$ for ${ }^{85} \mathrm{Rb}$ atoms for $\omega=2 \pi \times 17.5 \mathrm{~Hz}$ is $l=\sqrt{\hbar /(m \omega)}=25905 \AA$. One unit of time $t$ of Eq. (3) is $1 / \omega$ or $0.009095 \mathrm{~s}$. They prepared a stable ${ }^{85} \mathrm{Rb}$ condensate of $N_{0}=16000$ atoms with scattering length $a=a_{\text {initial }}=7 a_{0}, a_{0}=0.5292 \AA$, such that the initial $n=2.288$. Then during an interval of time $0.1 \mathrm{~ms}$ the scattering length was ramped to $a=a_{\text {collapse }}=-30 a_{0}$ such that final $n=-9.805$. The final condensate is strongly attractive and unstable and undergoes a sequence of collapse and explosion.

The sequence of collapse in many theoretical studies has been explained by introducing an absorptive imaginary three-body quintic interaction term of strength $\xi$ responsible for recombination loss. Consequently Eq. (3) becomes 6 - 8

$$
\begin{gathered}
{\left[-i \frac{\partial}{\partial t}-\frac{\partial^{2}}{\partial x^{2}}+\frac{1}{x} \frac{\partial}{\partial x}-\frac{\partial^{2}}{\partial y^{2}}+\frac{1}{4}\left(x^{2}+\lambda^{2} y^{2}-\frac{4}{x^{2}}\right)+\right.} \\
\left.8 \sqrt{2} \pi n\left|\frac{\varphi(x, y ; t)}{x}\right|^{2}-i \xi n^{2}\left|\frac{\varphi(x, y ; t)}{x}\right|^{4}\right] \varphi(x, y ; t)=0 .
\end{gathered}
$$

There are many ways to account for the loss mechanism [6.7]. It is quite impossible to include them all in a self consistent fashion. Here we simulate the loss via the most important quintic three-body term with parameter $\xi$. We use this single parameter to reproduce the experiment at JILA [15. For $\xi \neq 0$ in Eq. (7), $\mathcal{N}_{\text {norm }} \neq 1$.

We solve the GP equation (7) numerically by time iteration with a given initial solution. In the time-evolution of the GP equation the radial and axial variables are dealt with in independent steps. For this purpose we discretize it using time step $\Delta=0.001$ and space step 0.1 for both $x$ and $y$ spanning $x$ from 0 to 15 and $y$ from -25 to 25 . This domain of space was sufficient to encompass the whole condensate wave function even during and after explosion and collapse. The preparation of the initial repulsive wave function is now a routine job and was done by increasing the nonlinearity $n$ of the GP equation (7) by 0.0001 in each time step $\Delta$ during time iteration starting with the known harmonic oscillator solution of Eq. (7) for $n=\xi=0$ [16]. The initial value of $n(=2.288)$ was attained after 22880 time steps. The nonlinearity $n$ is then ramped from 2.288 to -9.805 in $0.1 \mathrm{~ms}$. As one unit of dimensionless time $t$ is $0.009095 \mathrm{~s}$, $0.1 \mathrm{~ms}$ corresponds to 11 steps of time $\Delta$. In the present simulation, $n$ is ramped from 2.288 to -9.805 in the GP equation by equal amount in 11 steps. The absorptive term $\xi$ was set equal to zero during above time iteration. Now the system is prepared for the simulation of collapse and explosion.

For the remaining simulation the nonlinear term is maintained constant and a nonzero value of $\xi$ is chosen. The time-evolution of the GP equation is continued as a function of time $t=\tau_{\text {evolve }}$ starting at 0 . Because of the nonzero dissipative term $\xi$, the normalization (4) is no longer maintained, and the number of remaining atoms $N$ in the condensate is given by $N=N_{0} \mathcal{N}_{\text {norm }}$, where $N_{0}$ is the initial number. The time-evolution is continued using time step $\Delta=0.001$. After a small experimentation it is found that $\xi=3.12$ fits the data of the experiment at JILA for $a_{\text {collapse }}=-30 a_{0}-$ their Fig. 1(b). This value of $\xi$ was used in all simulations reported here. The remaining number of atoms vs. time is plotted in Fig. 1.

It is pertinent to compare this value of $\xi(=3.12)$ with the experimental measurement of three-body loss rate on ${ }^{85} \mathrm{Rb}[23]$. For that, we need to restore the proper dimensions in the three-body term of Eq. (7) by rewriting it as $i \xi(a N / l)^{2}|\psi|^{4} l^{6} / 8$ and equating it to the convensional form $i K_{3} N^{2}|\psi|^{4} / 2 \omega$, where $K_{3}=\left(4.24_{-0.29}^{+0.70} \pm 0.85\right) \times$ $10^{-25} \mathrm{~cm}^{6} / \mathrm{s}$ is the experimental rate [23]. From this we find $K_{3}=\xi a^{2} l^{4} \omega / 4$. Under experimental condition of an external magnetic field of 250 gauss on ${ }^{85} \mathrm{Rb}$ [23] the scattering length was $a \sim-350 a_{0}$. Consequently, the present value of $\xi(=3.12)$ corresponds to $K_{3} \simeq 13 \times 10^{-25}$ $\mathrm{cm}^{6} / \mathrm{s}$, about three times larger than the experimental 7) value above.

In the experiment at JILA 15] it was observed that the strongly attractive condensate after preparation remains stable with a constant number of atoms for an interval of time $t_{\text {collapse }}$. This behavior is physically expected. Immediately after the jump in scattering length from $7 a_{0}$ to $-30 a_{0}$, the attractive condensate shrinks in size during $t_{\text {collapse }}$ until the central density increases to a maximum. Then the absorptive three-body term takes full control to initiate the sequence of collapse and explosion. Consequently, the number of atoms remains constant for $\tau_{\text {evolve }}<t$ collapse. The present result (full line) also shows a similar behavior. However, in this simulation the absorptive term is operative from $\tau_{\text {evolve }}=0$ and the atom number decreases right from beginning, albeit 
at a much smaller rate for $\tau_{\text {evolve }}<t$ collapse

Donley et al. 15] repeated their experiment with different values of final scattering length. Using the same value of $\xi(=3.12)$, we also repeated our calculation with the final scattering lengths: $a_{\text {collapse }}=-6.7 a_{0}$ and $-250 a_{0}$. These results are also plotted in Fig. 1. The initial delay $t_{\text {collapse }}$ in starting the explosion is large for small $\left|a_{\text {collapse }}\right|$ as we see in Fig. 1. After the initial delay, the decay rates of the number of atoms are quite similar as observed in the experiment. Similar effect was observed in the experiment for an initial condensate of 6000 atoms as shown in their Fig. 2.

After a sequence of collapse and explosion, Donley et al. [15] observed a "remnant" condensate of $N_{\text {remnant }}$ atoms at large times containing a certain fraction of initial $N_{0}$ atoms. Experimentally, the fraction of atoms that went into the remnant decreased with $\left|a_{\text {collapse }}\right|$ and was $\sim 40 \%$ for $\left|a_{\text {collapse }}\right|<10 a_{0}$ and was $\sim 10 \%$ $\left|a_{\text {collapse }}\right|>100 a_{0}$. Figure 1 also shows this behavior. We repeated the simulation of Fig. 1 with $N_{0}=6000$ where we also found a similar behavior. The number in the remnant condensate could be much larger than $N_{\mathrm{cr}}$.

The above evolution of the condensate after the jump in scattering length to $-30 a_{0}$ for $N_{0}=16000$ can be understood from a study of the wave function and we display the central part of the wave function in Fig. 2 for $\tau_{\text {evolve }}=0,3.5,4$, and $10 \mathrm{~ms}$. The wave function immediately after jump at time $\tau_{\text {evolve }}=0$ is essentially the same as that before the jump at $-0.1 \mathrm{~ms}$. There is not enough time for the wave function to get modified at $\tau_{\text {evolve }}=0$. From Fig. 2 we find that at $3.5 \mathrm{~ms}$ the wave function is only slighty narrower than at $0 \mathrm{~ms}$ but still smooth and has not yet collapsed significantly. As $\tau_{\text {evolve }}$ increases, the wave function contracts further and the explosion starts. At $4 \mathrm{~ms}$ some spikes have appeared in the wave function showing the beginning of explosions and loss. From the study of the wavefunctions we find that the explosions start at $\tau_{\text {evolve }}=t_{\text {collapse }} \simeq 3.7 \mathrm{~ms}$ in agreement with the experiment at JILA. We also find that at $3.7 \mathrm{~ms}$ before the loss began the bulk BEC did not contract dramatically as also observed in the experiment. In numerical simulation for this case we find that at $\tau_{\text {evolve }}=0, x_{\mathrm{rms}}=2.98 \mu \mathrm{m}$ and $y_{\mathrm{rms}}=4.21 \mu \mathrm{m}$ and at $\tau_{\text {evolve }}=3.7 \mathrm{~ns}, x_{\mathrm{rms}}=2.53 \mu \mathrm{m}$ and $y \mathrm{rms}=4.10 \mu \mathrm{m}$. From Fig. 2 we see that at $10 \mathrm{~ms}$ the wave function is very spiky corresponding to many violent ongoing explosions.

Donley et al. 115 also observed that the remnant condensate oscillated in a highly excited collective state with approximate frequencies $2 \nu$ axial and $2 \nu$ radial being predominantly excited. The measured frequencies were $13.6(6) \mathrm{Hz}$ and $33.4(3) \mathrm{Hz}$. To find if this behavior emerges from the present simulation we plot in Fig. 3 sizes $x_{\mathrm{rms}}$ and $y_{\mathrm{rms}}$ vs. time for the condensate after the jump in the scattering length to $-30 a_{0}$ from $7 a_{0}$ for $N_{0}=16000$. Excluding the first $20 \mathrm{~ms}$ when the remnant condensate is being formed, we find a periodic oscillation in $x_{\mathrm{rms}}$ and $y_{\mathrm{rms}}$ with frequencies $34 \mathrm{~Hz}$ and $13.6 \mathrm{~Hz}$, respectively. In addition, for $x_{\mathrm{rms}}$, superposed on 34 $\mathrm{Hz}$ we note a prominent frequency of about $3.5 \mathrm{~Hz}$, e.g., $0.2 \nu$ radial

Though we have explained some aspects of the experiment at JILA, there are certain detailed features that have not been obtained in this Letter. Donley et al. 15 have classified the emitted atoms in three categories: burst, jet and missing atoms. Detailed study of the wave function is needed to identify the jet atoms that appear possibly from the spikes in the wave function when the collapse is suddenly interrupted. The jets were also not always found to be symmetric about the condensate axis [15]. This makes an axially symmetric mean-field model as used in this Letter inappropriate for a description of the jet atoms. Moreover a clear-cut distinction between the burst and missing atoms emitted during explosions seems to be difficult in the present model. Also because of the missing (undetected) atoms it is difficult to predict the energy distribution of the burst atoms in a mean-field analysis which will only yield the total energy distribution of the burst plus missing atoms. A careful analysis of the energy of the emitted atoms is required for explaining these detailed features and would be a welcome future theoretical investigation.

In conclusion, we have employed a numerical simulation based on the accurate solution [16] of the mean-field Gross-Pitaevskii equation with a cylindrical trap to study the dynamics of collapse and explosion as observed in the recent experiment at JILA [15]. In the GP equation we include a quintic three-body nonlinear recombination loss term which accounts for the decay of the strongly attractive condensate. The results of the present simulation accounts for the essential features of that xperiment. We find that the exact inclusion of the asymmetry parameter $\lambda$ in the simulation is essential for a proper description of the experiment. In the experiment at JILA a strongly attractive ${ }^{85} \mathrm{Rb}$ condensate was prepared by ramping the scattering length to a large negative value and the subsequent decay of the condensate was measured. We have been able to understand the following features of this dynamics from the present numerical simulation: (1) The condensate undergoes a sequence of collapse and explosion and finally stabilizes to a remnant condensate at large times containing about $\sim 10 \%-40 \%$ of the initial number $N_{0}$. The number in the remnant condensate can be larger than the critical number for collapse $N_{\mathrm{cr}}$ for the same atomic interaction. (2) Both in the experiment and our simulation the remnant condensate executes radial and axial oscillations in a highly excited collective state for a long time with frequencies $2 \nu_{\text {radial }}$ and $2 \nu_{\text {axial }}$. (3) The rate of decay of the number of particles is reasonably constant independent of $N_{0}$ and $a_{\text {collapse }}$ (4) After the sudden change in the scattering length to a large negative value, the condensate needs an interval of time $t_{\text {collapse }}$ before it experiences loss via explosions. Consequently, 
the decay starts after the interval of time $t_{\text {collapse }}$.

The investigation is supported in part by the CNPq and FAPESP of Brazil.

[1] J. R. Ensher, D. S. Jin, M. R. Matthews, C. E. Wieman, E. A. Cornell, Phys. Rev. Lett. 77 (1996) 4984.

[2] K. B. Dadic, M. O. Mewes, M. R. Andrews, N. J. van Druten, D. S. Durfee, D. M. Kurn, W. Ketterle, Phys. Rev. Lett. 75 (1995) 3969.

[3] D. G. Fried, T. C. Killian, L. Willmann, D. Landhuis, S. C. Moss, D. Kleppner, T. J. Greytak, Phys. Rev. Lett. 81 (1998) 3811.

[4] J. M. Gerton, D. Strekalov, I. Prodan, R. G. Hulet, Nature 408 (2001) 692.

[5] C. C. Bradley, C. A. Sackett, R. G. Hulet, Phys. Rev. Lett. 78 (1997) 985.

[6] H. Saito, M.Ueda, Phys. Rev. Lett. 86 (2001) 1406.

[7] R. A. Duine, H. T. C. Stoof, Phys. Rev. Lett. 86 (2001) 2204.

[8] Y. Kagan, A. E. Muryshev, G. V. Shlyapnikov, Phys. Rev. Lett. 81 (1998) 933.

[9] A. Gammal, T. Frederico, L. Tomio, Ph. Chomaz, Phys. Rev. A 61 (2000) 051602.

[10] M. Ueda, K. Huang, Phys. Rev. A 61 (1999) 043601.

[11] F. Dalfovo, S. Giorgini, L. P. Pitaevskii, S. Stringari, Rev. Mod. Phys. 71 (1999) 463.

[12] S. Inouye, M. R. Andrews, J. Stenger, H. J. Miesner, D. M. Stamper-Kurn, W. Ketterle, Nature 392 (1998) 151.

[13] J. L. Roberts, N. R. Claussen, S. L. Cornish, E. A. Donley, E. A. Cornell, C. E. Wieman, Phys. Rev. Lett. 86 (2001) 4211.

[14] S. L. Cornish, N. R. Claussen, J. L. Roberts, E. A. Cornell, C. E. Wieman, Phys. Rev. Lett. 85 (2000) 1795.

[15] E. A. Donley, N. R. Claussen, S. L. Cornish, J. L. Roberts, E. A. Cornell, C. E. Wieman, Nature 412 (2001) 295.

[16] S. K. Adhikari, Phys. Rev. E 65 (2002) 016703.

[17] S. K. Adhikari, Phys. Rev. E 62 (2000) 2937.

[18] S. K. Adhikari, Phys. Rev. E 63, (2001) 056704.

[19] S. K. Adhikari, Phys. Rev. A 63 (2001) 043611.

[20] M. Holland, J. Cooper, Phys. Rev. A 53 (1996) R1954.

[21] A. Gammal, T. Frederico, L. Tomio, Phys. Rev. A 64 (2001) 055602.

[22] F. Dalfovo, S. Stringari, Phys. Rev. A 53 (1996) 2477.

[23] J. L. Roberts, N. R. Claussen, S. L. Cornish, C. E. Wieman, Phys. Rev. Lett. 85 (2000) 728.

\section{Figure Caption:}

1. Number of remaining atoms in the ${ }^{85} \mathrm{Rb}$ condensate of 16000 atoms after ramping the scattering length from $a_{\text {initial }}=7 a_{0}$ to $a_{\text {collapse }}=-6.7 a_{0},-30 a_{0}$ and $-250 a_{0}$ in $0.1 \mathrm{~ms}$ as a function of evolution time $\tau_{\text {evolve }}$ in ms. The solid circles 15 are from experiment with $a_{\text {collapse }}=-30 a_{0}$ and the theoretical curves are labeled by the values of $a_{\text {collapse }}$.

2. The central part of the dimensionless wave function $|\phi(x, y)| \equiv|\varphi(x, y) / x|$ of the condensate on $0.1 \times 0.1$ grid after the jump in the scattering length of a BEC of $16000{ }^{85} \mathrm{Rb}$ atoms from $7 a_{0}$ to $a_{\text {collapse }}=-30 a_{0}$ at times $\tau_{\text {evolve }}=0,3.5 \mathrm{~ms}, 4 \mathrm{~ms}$, and $10 \mathrm{~ms}$.

3. The dimensionless rms sizes $x_{\mathrm{rms}}$ (full line) and yrms (dashed line) expressed in units of $l / \sqrt{2}$ after the jump in the scattering length of a BEC of $16000{ }^{85} \mathrm{Rb}$ atoms from $a_{\text {initial }}=7 a_{0}$ to $a_{\text {collapse }}=-30 a_{0}$ as functions of time $\tau_{\text {evolve }}$. 


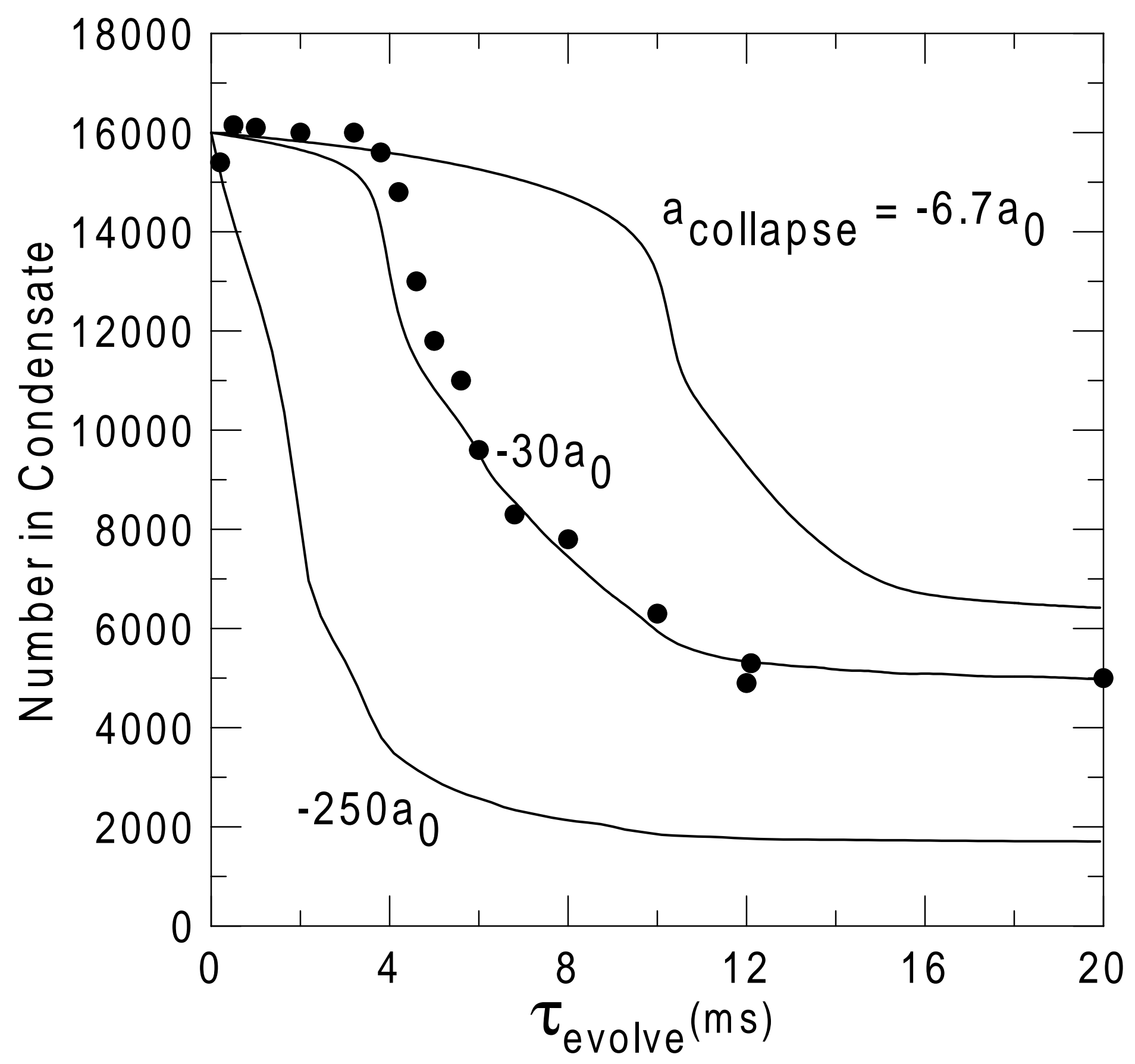



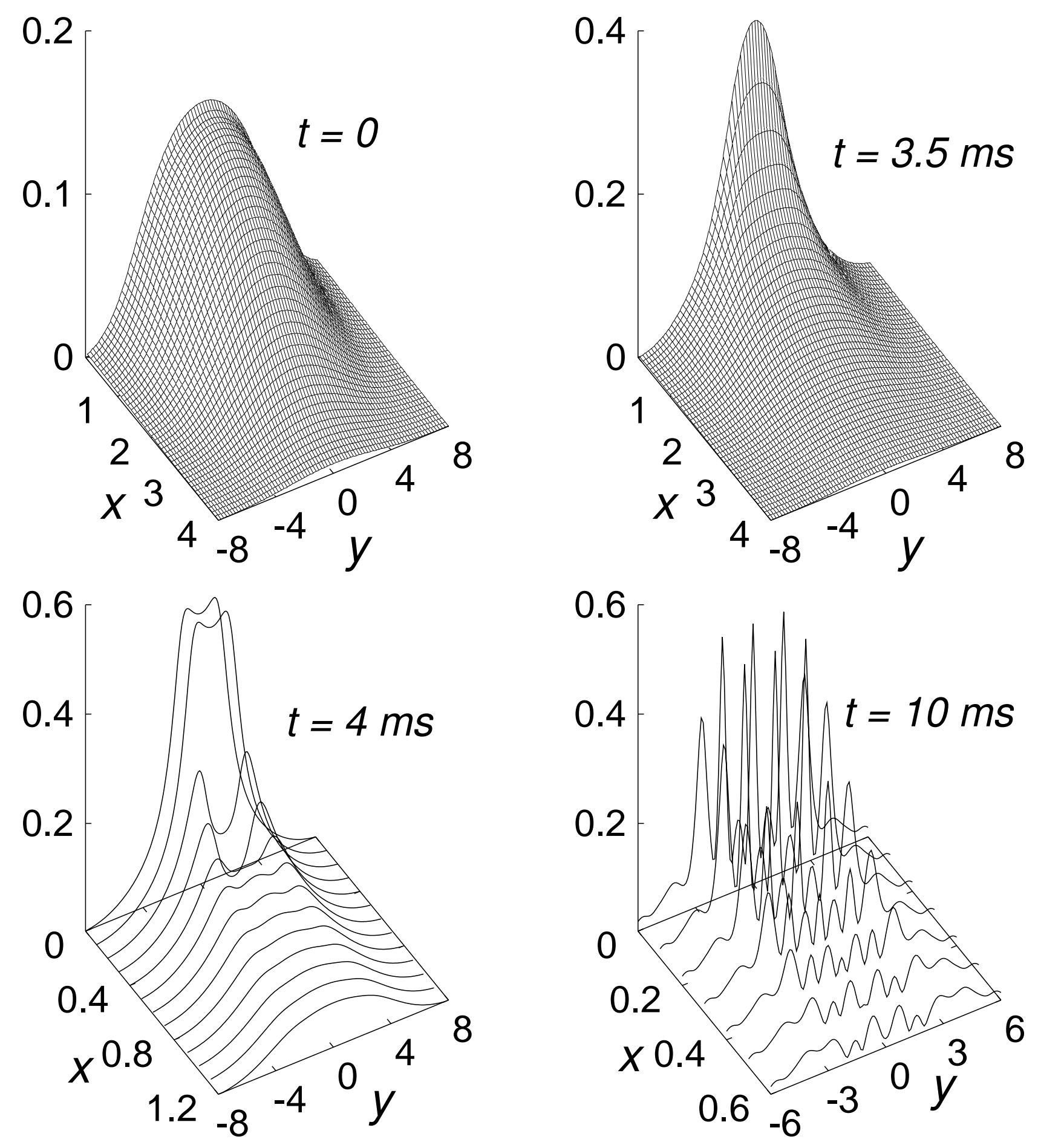


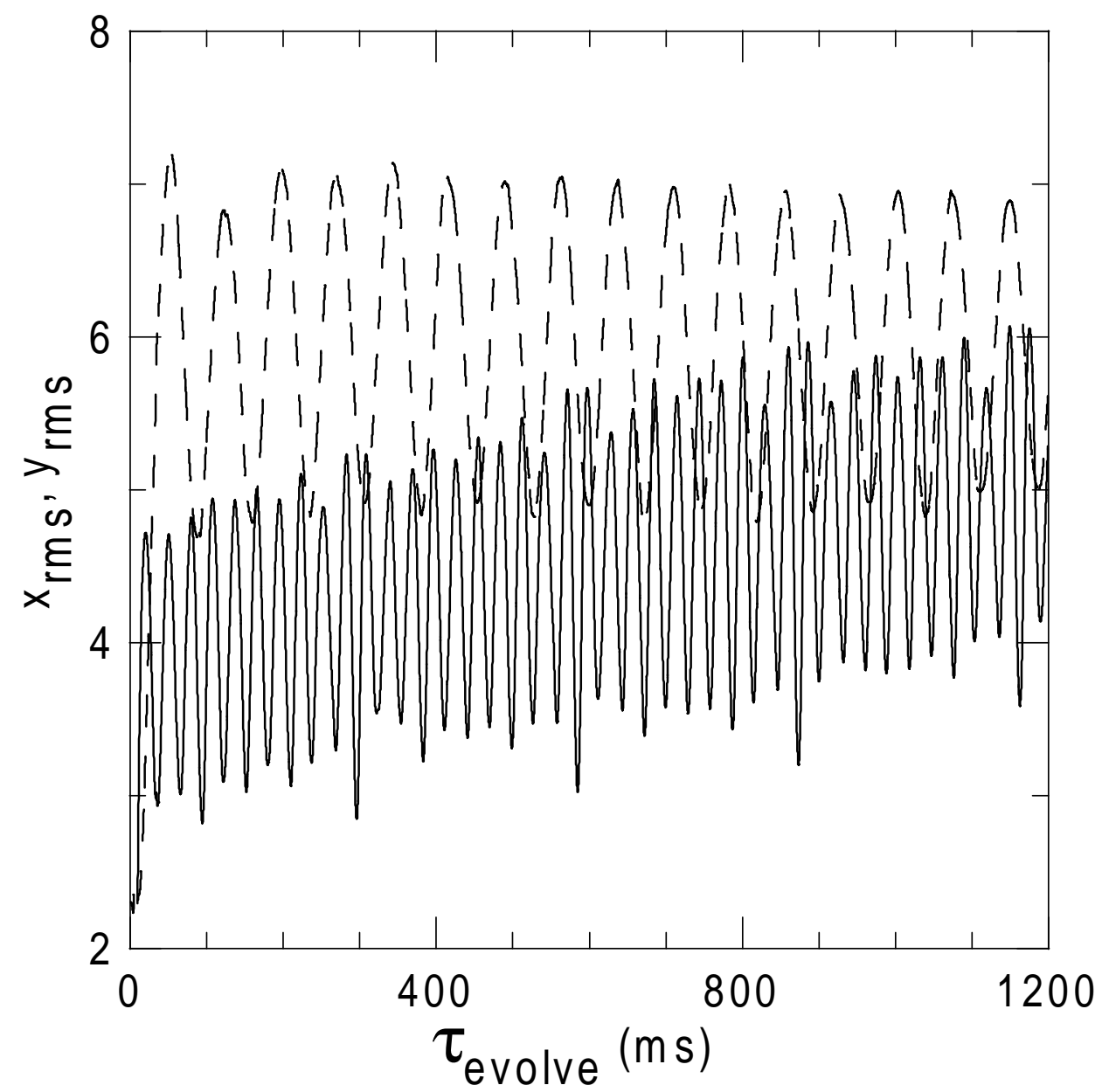

